\title{
SIMULTANEOUS STRAIN AND TEMPERATURE MEASUREMENT USING COMBINED FIBER AND FIBER BRAGG GRATING SENSORS
}

\author{
Hedi Bellil \\ Institut Superieur des Etudes Technologiques en Communications de Tunis, Tunis \\ hedi.bellil@isetr.rnu.tn \\ Mustafa A.G. Abushagur \\ The University of Alabama in Huntsville Department of Electrical and Computer Engineering, \\ Huntsville, Alabama, United States
}

DOI: $10.36724 / 2664-066 X-202 \mid-7-3-6-10$

\begin{abstract}
We propose here a new technique for simultaneous strain and temperature measurement using a Fiber Bragg Grating sensors. This technique employs an interferometric detection using two Fiber Bragg Gratings. A fiber Bragg grating is used as a reference while another fiber Bragg grating and a length of a bare fiber are used as the two sensing elements. As the temperature and strain change, the length of the sensing elements, (Fiber and Fiber Bragg Grating) change. These changes result in a phase and wavelength shifts. To measure these effects we use a Folded Mach Zhender interferometer, and a detection system made out of two photodiodes and data processing system. The sensitivity and dynamic range are analyzed and presented.
\end{abstract}

KEYWORDS: Fiber Bragg Grating sensors, optical fiber sensors, wavelength detection 


\section{Introduction}

Optical fiber sensors became devices of choice for many applications over the years. Fiber Bragg Gratings (FBG) are of interest for a variety of sensing applications [1] and in the development of novel all-fiber optical devices such as stop-band and band-pass filters [2], wavelength taps and Fabry-Perot filters [3], strain sensing in smart structures and composite materials [4].FBG sensors are currently the focus of considerable research effort. They are particularly well suited for measuring strain in smart structures [5], and temperature [6]. However, undesirable temperature (or strain) sensitivity of the fiber grating sensor may complicate its application as strain (or temperature) gauge.

As the strain and the temperature are tightly dependent of each other, it is difficult to differentiate between the effects of changes in them. A variety of sensors were proposed to measure both quantities simultaneously. Thermal effect can be well compensated using a pair of fiber gratings in a certain configuration [7]. Two different types of photogenerated fiber grating, namely, a fiber Bragg grating and a fiber polarization-rocking filter [8], the method relies on the different dependencies of the fiber refractive index and birefringence on strain and temperature. It has been suggested that temperature can be measured and compensated using a second grating element contained within a different material and placed in series with the first grating element [9]. The problem of discriminating between strain and temperature has been tackled by use of two Bragg gratings written on the same fiber and operating at different wavelengths [10].

It has been demonstrated that the use of interferometric detection of the phase change or the wavelength shift of Fiber Bragg Grating sensors can yield very high sensitivity of grating temperature or strain. One remaining issue is the separation of the strain-induced wavelength shift from that induced by temperature changes. In dual measurand techniques, strain and temperature are determined from measurement on a "pair of sensors" which are both sensitive to strain and temperature, but have different characteristics.

In this paper, we suggest a novel technique to resolve these two effects dependencies.

We propose here a new technique for simultaneous strain and temperature measurement using a Fiber Bragg Grating sensors. This technique employs an interferometric detection using two Fiber Bragg Gratings. A fiber Bragg grating is used as a reference while another fiber Bragg grating and a length of a bare fiber are used as the two sensing elements.

As the temperature and strain change, the length of the sensing elements, (fiber and FBG), change. These changes result in a phase and wavelength shifts. To measure these effects we use a Folded Mach Zhender (FMZ) interferometer, and a detection system made out of two photodiodes and data processing system. The sensitivity and dynamic range are analyzed and presented.

\section{Theoretical considerations}

In this paper, we present a method by which both temperature and strain can be unambiguously determined when they are applied onto the fiber. The FBGs interferometer is used to measure simultaneously these effects by using two identical gratings at room temperature and without strain applied to them.

The setup arrangement used to simultaneously measure strain and temperature using FBGs is shown in Fig.1. Light from a broadband source is coupled into an interferometer with two arms using two FBGs, one arm is used as reference and the second (composed of a grating and a fiber) is used as a sensing arm. Light reaches from the broadband source and partially reflected by the FBGs. The light reflected from FBG1 and FBG2 is at wavelengths $\lambda_{1}$ and $\lambda_{2}$, respectively. In the case of no change in either temperature or strain, $\lambda_{1}$ and $\lambda_{2}$, will be identical.

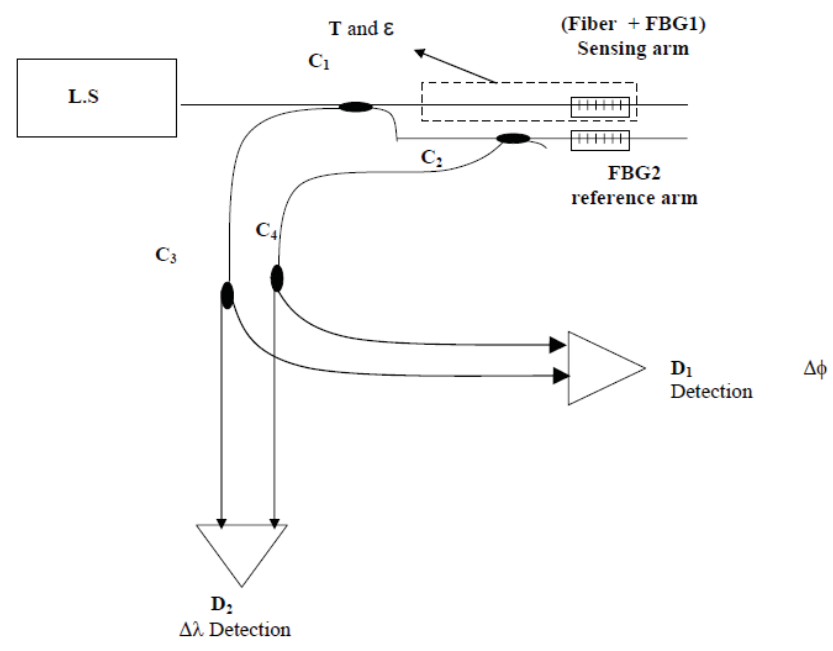

Fig. 1. Fiber Bragg Grating sensor system with sensing (Fiber + Grating) and reference grating elements, and interferometric phase change and wavelength detection

Since the temperature and strain, affect the sensing arm (fiber and grating), this leads to a change in the properties of the fiber and grating, this results in a phase and wavelength shift. If the temperature and/or strain change around the sensing arm the $\lambda_{1}$ will shift accordingly. The waves reflected from both Bragg gratings are combined by the couplers $\mathrm{C}_{1}$ and $\mathrm{C}_{2}$, and conveniently separated by using two couplers $\mathrm{C}_{3}$ and $\mathrm{C}_{4}$. Light from $\mathrm{C}_{3}$ and $\mathrm{C}_{4}$ was split and four waves were launched on two detectors $\mathrm{D}_{1}$ and $\mathrm{D}_{2} \cdot \mathrm{D}_{1}$ is used to detect a phase change $\Delta \phi$ and $\mathrm{D}_{2}$ is used to detect the Bragg wavelength shift, $\Delta \lambda_{\mathrm{b}}=\left|\lambda_{2}-\lambda_{1}\right|$.

The corresponding phase change sensitivity due to the temperature and strain effects on the bare fiber is given by, 


$$
\begin{aligned}
& \Delta \phi / \phi=(1 / \mathrm{n} \partial \mathrm{n} / \partial \mathrm{T}+1 / \mathrm{L} \partial \mathrm{L} / \mathrm{L}) \Delta \mathrm{T}+ \\
& (1 / \mathrm{n} \partial \mathrm{n} / \partial \varepsilon+1 / \mathrm{L} \partial \mathrm{L} / \partial \varepsilon) \Delta \varepsilon
\end{aligned}
$$

$$
\begin{aligned}
& \Delta \phi / \phi=\left(1 / \mathrm{n} \partial \mathrm{n} / \partial \mathrm{T}+\alpha_{\mathrm{T}}\right) \Delta \mathrm{T}+ \\
& +\left[1-\mathrm{n}^{2} / 2\left(\mathrm{P}_{11}+2 \mathrm{P}_{12}\right)\right] \Delta \varepsilon
\end{aligned}
$$

where $n$ and $L$ are the refractive index and the Length of the fiber, respectively, $\alpha \mathrm{T}$ is the coefficient of thermal expansion, $1 / \mathrm{n} \partial \mathrm{n} / \partial \mathrm{T}$ is the thermo-optic coefficient, $\Delta \mathrm{T}$ and $\Delta \varepsilon$ are the temperature and strain applied on the fiber respectively, and $\mathrm{P}_{11}$ and $\mathrm{P}_{12}$ are the Pockel's coefficients.

The shift in the Bragg wavelength sensitivity due to the temperature and strain effects on the FBG can be expressed using:

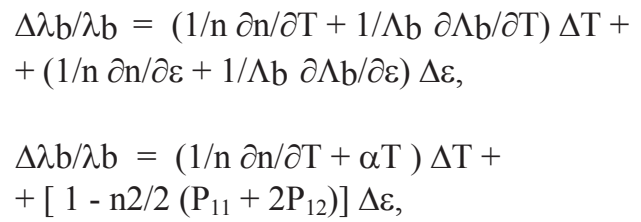

where $\Lambda_{\mathrm{b}}$ is the period of the grating.

Eqs. (1) and (2) can be reduced to a set of linear equations in $\Delta \phi$ and $\Delta \lambda_{\mathrm{b}}$ for any applied strain and temperature which is given by:

$$
\Delta \phi / \phi=\mathrm{A} \Delta \mathrm{T}+\mathrm{B} \Delta \varepsilon
$$

and

$$
\Delta \lambda_{\mathrm{b}} / \lambda_{\mathrm{b}}=\mathrm{C} \Delta \mathrm{T}+\mathrm{D} \Delta \varepsilon
$$

where the temperature change is in ${ }^{\circ} \mathrm{C}$ and the strain is in $\mu$ strain, A, B, C, and D are constants which depend on thermo-optic, thermal expansion, and Pockel's coefficients related to the Fiber material and the FBG.

The constants have a numerical values of $[11,12]$ :

$$
\begin{aligned}
& \mathrm{A} \approx 7.42 \times 10^{-6}\left({ }^{\circ} \mathrm{C}\right)^{-1}, \mathrm{~B} \approx 0.52 \times 10^{-6}(\mu \text { strain })^{-1} \\
& \mathrm{C} \approx 7.50 \times 10^{-6}\left({ }^{\circ} \mathrm{C}\right)^{-1}, \quad \mathrm{D} \approx 0.78 \times 10^{-6}(\mu \text { strain })^{-1}
\end{aligned}
$$

\section{Numerical analysis}

To demonstrate the sensor sensitivity to the temperature and strain, we need to calculate $\Delta \phi$ and $\Delta \lambda \mathrm{b}$, a set of known strains and temperatures were applied to the sensor.

In this numerical analysis of the linear system, Eq.(3) we examine the phase and wavelength change as a function of one of the variables while fixing the other. The sensitivity, $\Delta \lambda_{\mathrm{b}} / \lambda \mathrm{b}$, obtained by our method is around of $0.78 \times 10^{-6}$ ( $\mu$ strain $)^{-1}$. For $\lambda b=1550 \mathrm{~nm}$, we obtain a wavelength shift $\Delta \lambda \mathrm{b} \cong 0.0012 \mathrm{~nm} / \mu$ strain.
The phase shift sensitivity, $\Delta \phi / \phi$, is about $0.52 \times 10^{-6}$ ( $\mu$ strain) $^{-1}$. If the sensing fiber is $0.25 \mathrm{~m}$ long, the phase shift $\Delta \phi \cong 1,53 \mathrm{rad} / \mu$ strain. The sensitivity can be altered by choosing different lengths of the fiber.

We obtain a wavelength sensitivity about $7.5 \times 10^{-6}\left({ }^{\circ} \mathrm{C}\right)^{-1}$. The wavelength shift for the same $\lambda \mathrm{b}$ is

$$
\Delta \lambda \mathrm{b} \cong 0.0011 \mathrm{~nm}\left({ }^{\circ} \mathrm{C}\right)^{-1},
$$

and a phase change $\Delta \phi \cong 1.5 \mathrm{rad} /{ }^{\circ} \mathrm{C}$.

The sensitivity can be altered by changing the characteristics of the FBG.

\section{Detection system:}

The sensor is used for simultaneously measuring the wavelength shift, $\Delta \lambda \mathrm{b}$, of the Bragg wavelength and the phase change, $\Delta \phi$, between two waves. Therefore, double detection was used. The output optical wave from the interferometer can be represented by the electric fields, reflected from the FBGs. These two optical waves can be represented by,

$$
E_{1}=E_{01} e^{j(\omega t+\phi)} \text { and } E_{2}=E_{02} j^{(\omega t+\phi 2)}
$$

where $\phi_{1}$ and $\phi_{2}$ are the relative phases of the two waves, and $\omega$ is the angular frequency.

The output waves are detected as a corresponding current intensity given by

$$
\mathrm{I}=\mathrm{E} \cdot \mathrm{E}^{*} \text {. }
$$

where $\mathrm{E}=\mathrm{E}_{1}+\mathrm{E}_{2}$ is the resultant wave, and * is the complex conjugate, the current is given by,

$$
\mathrm{I}=\mathrm{I}_{1}+\mathrm{I}_{2}+\left(\mathrm{e}^{\mathrm{i} \Delta \phi}+\mathrm{e}^{-\mathrm{i} \Delta \phi}\right) \sqrt{ } \mathrm{I}_{1} \mathrm{I}_{2},
$$

for the case where, $I_{0}=I_{1}=I_{2}$, I becomes

$$
\mathrm{I}=2 \mathrm{I}_{0}[1+\cos .(\Delta \phi)]
$$

Eq. (5c), provides the relationship between the output current on the detector D1 and the phase change between the two waves which are reflected from the FBGs. Fig.2 represents the plot of the output current as a function of the phase change $\Delta \phi$ of the detector D1. Once the $\Delta \phi$ is known, we have to detect the wavelength shift $\Delta \lambda \mathrm{b}$. For this measurement, we use a heterodyne detection, and we calculate the wavelength shifts $\Delta \lambda \mathrm{b}$ of the reflected light, induced by the temperature and strain changes in the sensing elements.

The output optical wave from the interferometer can be represented by the electric fields reflected from the FBGs and detected by the detector $\mathrm{D}_{2}$. 


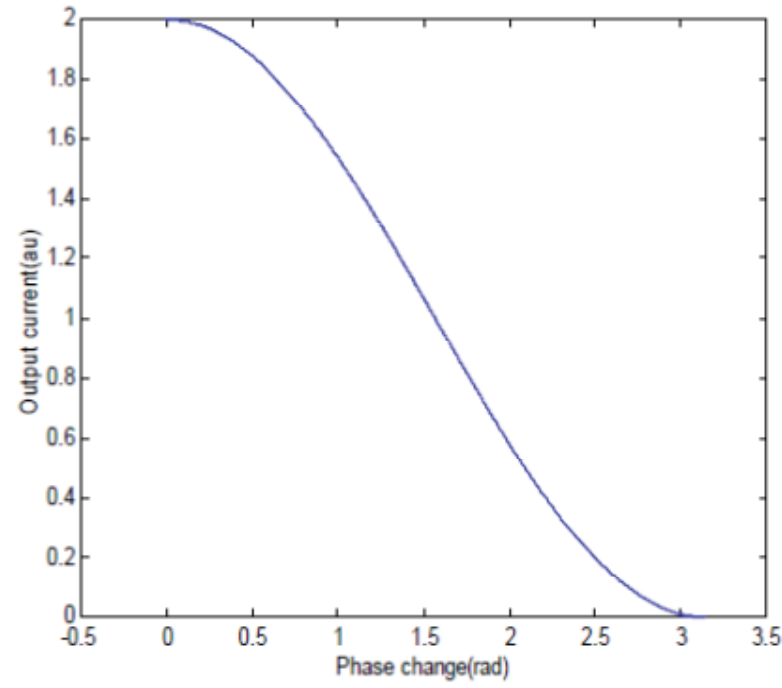

Fig. 2. Output signal obtained on the detector $D_{1}$ v.s. the phase change $\Delta \phi$

These two optical waves are given by

$$
\begin{aligned}
& \Psi_{1}=A_{1} \cos \left(\omega_{1} t+\phi_{1}\right) \text { and } \\
& \Psi_{2}=A_{2} \cos \left(\omega_{2} t+\phi_{2}\right)
\end{aligned}
$$

where $\omega_{1}$ and $\omega_{2}$ are the angular frequencies of the reflected signals from the reference and the sensing interferometer arms, respectively. $\phi_{1}$ and $\phi_{2}$ are the relative phases of the two signals $\left(\phi_{1}=\phi_{2}\right.$ and $\omega_{1}=\omega_{2}$, when temperature and strain effects are absent). $A_{1}$ and $\mathrm{A}_{2}$ are amplitudes of the optical waves.

The output waves were detected with a photodiode which acts as a square-law detector. The photo- current generated by the photodiode is given by

$$
\begin{aligned}
& \mathrm{I}(\mathrm{t}, \mathrm{T}, \varepsilon)=\mid \mathrm{A}_{1} \cos \left(\omega_{1} \mathrm{t}+\phi_{1}\right)+ \\
& +\left.\mathrm{A}_{2} \cos \left(\omega_{2} \mathrm{t}+\phi 2\right)\right|^{2} .
\end{aligned}
$$

In Eq.(7) we dropped the effect of the photodiode responsivity. The photodiode will act as a low-pass filter for the optical frequencies. Therefore, the output current will contain only signals with frequencies lower than that of the optical signal. Therefore the output current is given by:

$$
\mathrm{I}=\mathrm{A} \cos \left(\Delta \omega_{\mathrm{t}}+\Delta \phi\right),
$$

where

$$
\begin{aligned}
& \Delta \omega=\left(\omega_{2}-\omega_{1}\right), \\
& \Delta \phi=\left(\phi_{2}-\phi_{1}\right) .
\end{aligned}
$$

The amplitude $\mathrm{A}$ depends on $\mathrm{A}_{1}, \mathrm{~A}_{2}$ and the responsivity of the photodiode.

As the temperature and strain change, the frequency, $\Delta \omega$, and the phase, $\Delta \phi$, change accordingly. From Eq.(2b), $\Delta \omega$ can be expressed as a function of $\Delta \mathrm{T}$ and $\Delta \varepsilon$ by,

$$
\begin{aligned}
& \Delta \omega=\left(2 \pi \mathrm{C} / \lambda_{\mathrm{b}}\right)\left[\left(1 / \mathrm{n} \partial \mathrm{n} / \partial \mathrm{T}+1 / \Lambda_{\mathrm{b}} \partial \Lambda_{\mathrm{b}} / \Lambda_{\mathrm{b}}\right) \Delta \mathrm{T}+\right. \\
& \left.+\left(1 / \mathrm{n} \partial \mathrm{n} / \partial \varepsilon+1 / \Lambda_{\mathrm{b}} \partial \Lambda_{\mathrm{b}} / \partial \varepsilon\right) \Delta \varepsilon\right] .
\end{aligned}
$$

and $\Delta \phi$ is known from the Eq.(5b).

The instantaneous output current given by Eq.(7), is plotted as a function of temperature and strain as shown in Fig.(3). We have considered two cases for the strain by keeping temperature change constant, and for the temperature while the strain still constant.

In Fig. 3(a), the dynamic range is $17^{\circ} \mathrm{C}$ for the temperature and Fig.3(b) shows a dynamic range of 150 $\mu$ strains for the strain.

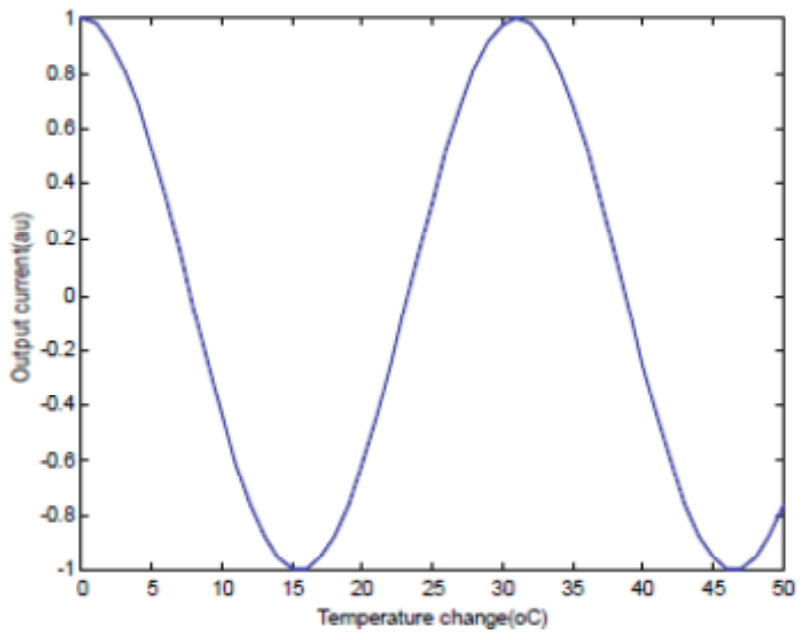

(a)

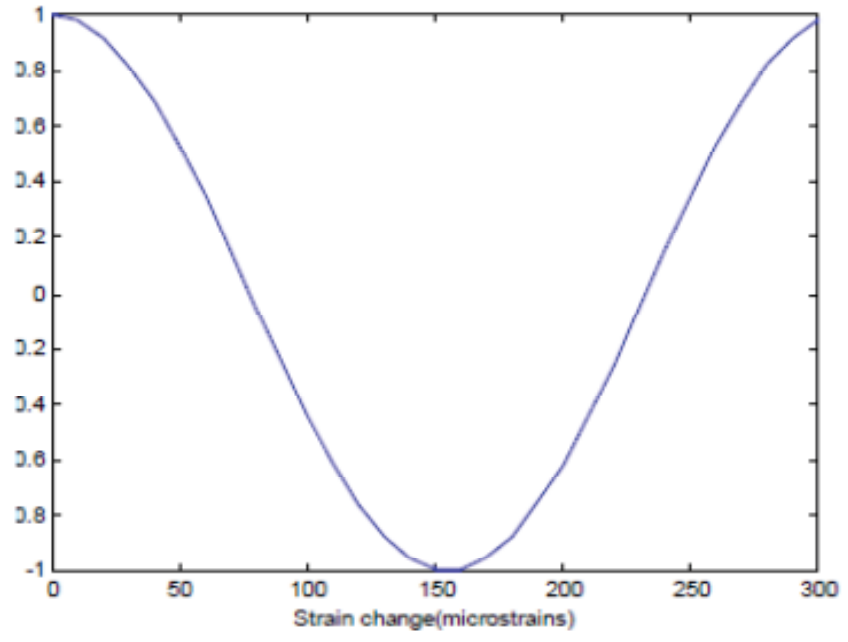

(b)

Fig. 3. The output current of detector $\mathrm{D}_{2}$ as a function of temperature and strain, $(\boldsymbol{a})$ strain is constant, $(\boldsymbol{b})$ temperature is constant 


\section{Conclusions}

In summary, we have introduced a fiber optic and FBG sensors that measures simultaneously the temperature and the strain changes in the environment surrounding the sensing arm. The sensitivity and dynamic range are flexible and can be controlled by choosing the length of the bare fiber as well as the characteristics, e.g. grating length and period, of the FBG.

\section{References}

[1] G. Meltz, W.W. Morey, and W.H. Glenn, "Formation of Bragg gratings in optical fibers by a transverse holographic method."Opt. Lett. , Vol. 14, pp. 823-825, 1989.

[2] W.W. Morey, "Distributed fiber grating sensors." Proc. OFS' 90, Sydney, Australia, Dec. 1990, pp. 285-288.

[3] W.W. Morey, J.R. Dunphy, and G. Meltz, "Multiplexing fiber Bragg grating sensors." Proc. Distributed and Multiplexed Fiber, Opt. Sensors, SPIE, Vol. 1586, Boston, Sept. 1991, pp. 216-224.

[4] R.M.. Measures, S.Melle and K.Liu, "Wavelength demodulation Bragg grating fiber optic sensing systems for addressing smart structure critical issues." Smart Mater. and Struct., Vol.1, pp. 36-44, 1992.
[5] W.W. Morey, G. Meltz, and W.H. Glenn, "Fiber Bragg Grating sensors." Proc. SPIE, Vol. 1169, 1989, pp. 98-107.

[6] A.D. Kersey, and T.A. Berkoff, "Fiber optic Bragg grating differential temperature sensor." IEEE Photon. Technol. Lett., Vol. 4, N0. 10, 1993, pp. 1183-1185.

[7] Heather J. Patrick and Sandeep T. Vohra, "Fiber Bragg Grating with long period fiber grating superstructure for simultaneous strain and temperature measurement." European Workshop on Optical Fiber Sensors, SPIE Vol.3483, 1998, pp. 264-267.

[8] Sotiris E. Kanellopoulos, Vicent A. Handerek, and Alan J. Rogers, "simultaneous strain and temperature sensing with photogenerated in-fiber gratings." Opt. Lett., Vol. 20, N0. 3, 1995, pp. 333-335.

[9] W. W. Morey, G. Meltz, and J. M. Weiss, "Evaluation of a fiber Bragg grating hydrostatic pressure sensor." Proc. OFS'8, Monterey, 1992, Postdeadline paper PD-4.

[10] M. G. Xu, J. L. Archambault, L. Reekie, and J. P. Dakin, "Discrimination between strain and temperature effects using dual-wavelength fiber grating sensors." Electron. Lett., 31, 1085, 1994.

[11] A.D.Kersey, M.A.Davis, H.J.Patrick, M.Leblanc, K.P.Koo, C.G.Askin, M.A.Putnam, and E.J.Friebele, "Fiber Grating Sensors." J. Lightwave Technol., Vol.15, No.8, 1997, pp.1442-1463.

[12] G.B.Hocker, "Fiber-optic sensing of pressure and temperature." Appl. Opt., Vol.18, No.9, 1979, pp.1445-1448. 